\title{
Tratamiento Térmico de Aceros de Bajo Carbono en Horno de Atmósfera Controlada
}

\author{
Fernando Franco* \\ José Harrinson Paz**
}

* M.Sc. Profesor - Esculea de Ingeniería de Materiales Facultad de Ingeniería - Universidad del Valle - Santiago de Cali, Colombia.

E-mail: ffrancoa@univalle.edu.co

** Ingeniero de Materiales - Esculea de Ingeniería de

Materiales - Facultad de Ingeniería - Universidad del Valle

- Santiago de Cali, Colombia.

e-mail:josehpaz@yahoo.es
Fecha de recepción: Septiembre 30 de 2004

Fecha de aprobación: Diciembre 17 de 2004

\section{RESUMEN}

Se presenta el proceso de desarrollo y evaluación de un generador de atmósfera endotérmica prototipo y un horno para tratamiento térmico de aceros en medio gaseoso realizado en la escuela de Ingeniería de Materiales de la Universidad del valle.

Se definieron los parámetros necesarios para la generación de la atmósfera endotérmica como son: la temperatura, relación aire/gas, presión de trabajo. Se escogió el propano comercial como gas generador y un catalizador de Alúmina-Míquel para propiciar la reacción. Se desarrolló y construyó el sistema generador y el equipo de tratamiento térmico necesario teniendo como base elementos disponibles en el laboratorio de tratamientos térmicos. 
La evaluación experimental del equipo desarrollado se hizo mediante el tratamiento térmico de temple de dos aceros normalizados tipo AISI 1018 y 1025 en una atmósfera protectora. Se realizaron análisis gravimétricos, metalográficos y de microdureza para determinar los cambios de microestructura y propiedades mecánicas y de profundidad de capa afectada en las muestras tratadas.

Se obtuvieron resultados positivos, abriéndose paso a un futuro mejoramiento del diseño e investigación complementaria de aplicación directa en la industria.

Palabras Clave: Tratamiento térmico, generador de atmósfera endotérmica, atmósferas controladas.

\section{ABSTRACT}

The process of development and experimental evaluation of an endothermic gas generator used for the thermal treatment of steels developed in the Materials Engineering School of the Universidad Del Valle is presented in this report.

As a starting point, the operative parameters required to the endothermic gas generation; such as, temperature, air/gas ratio, working pressure using commercial propane and nickel based catalizer to promote the reaction were selected. The required equipment was developed taking into account some elements already available in the laboratory of heat treatment.

Experimental testing was developed on low carbon normalized steels AISI 1018 and 1025 with a heat treatment of quenching. Gravimetric, and metallographic analysis, hardness tests and thickness measuring of affected layer were conducted on prepared specimens with very positive results, opening the route for future design developments and complementary research on controlled atmospheres to be directly applied in industry.
Key words: Heat treatment, endothermic gas generator, controlled atmospheres.

\section{INTRODUCCIÓN}

Los tratamientos térmicos son procesos comúnmente aplicados para mejorar las propiedades mecánicas en el procesamiento de los aceros para uso industrial. Su principio se basa en someter el material a ciclos de calentamiento, sostenimiento y enfriamiento que puede variar de acuerdo a las propiedades deseadas en el material. El oxigeno del aire o gases oxidantes, producen la oxidación de las capas superficiales con formación de cascarilla que envuelve todo el material y que, cuando se presenta en gran cantidad, se separa con relativa facilidad. Este óxido origina importantes pérdidas de material y da lugar a irregularidades superficiales y deficiente control de tolerancias dimensionales.

Otra reacción indeseable en un tratamiento térmico es la descarburación superficial, la cual es una pérdida del carbono de las zonas periféricas de los aceros, que se produce a altas temperaturas bajo la acción de determinadas mezclas gaseosas principalmente hidrógeno, oxigeno, dióxido de Carbono y vapor de agua. $(1,2)$

La introducción de "atmósferas protectoras" en los hornos de tratamiento térmico permite controlar estos procesos de oxidación, descarburación y carburación de los aceros.

Existe una variedad de atmósferas protectoras que ofrecen diferentes características de acuerdo al material a tratar. Uno de estos tipos de atmósferas es la atmósfera endotérmica, la cual puede ser usada en virtualmente todos los procesos de hornos que requieran fuertes condiciones reductoras. De utilidad especial en procesos industriales que involucran tratamiento térmico de temple, recocido, revenido y cementación en la cual se comportaría como protectora y carburante a la vez. $(1,2)$ 
Cuando un gas combustible arde sobre $1000^{\circ} \mathrm{C}$ con una pequeña cantidad de aire, menor a la requerida para producir combustión completa (relación de aire/gas 0.4 a 0.5) se genera una atmósfera endotérmica "Endogas", la cual consta de $\mathrm{CO}, \mathrm{CO}_{2}, \mathrm{M}_{2}, \mathrm{H}_{2} \mathrm{O}, \mathrm{H}_{2}$. En consecuencia el gas hidrocarburo y el aire son regulados en una proporción tal que el $\mathrm{O}_{2}$ sea el suficiente para formar $\mathrm{CO}$ y $\mathrm{H}_{2}$, sin exceso de $\mathrm{CO}_{2}$ y vapor de agua. La composición básica de una atmósfera endotérmica para el propano como gas base, se muestra en el diagrama de combustión de la figura 1. El nombre de atmósfera endotérmica proviene del hecho que para mantener la reacción es necesario calentamiento externo debido a la baja proporción aire/combustible (3).

La atmósfera endotérmica se prepara haciendo pasar el gas base o una mezcla de gas y aire por un catalizador colocado en el interior de unos tubos de acero refractario al calor, colocados dentro de un horno (retorta). Al calentarse el horno y elevarse la temperatura de los tubos, se produce la disociación del combustible en contacto con el catalizador de acuerdo con la reacción (1) y provoca una segunda reacción del carbono disociado con el oxigeno del aire para producir CO, según la reacción $(2) .(4,5,6)$.

$$
\begin{aligned}
& \mathrm{C}_{3} \mathrm{H}_{8} \rightarrow 4 \mathrm{H}_{2}+3 \mathrm{C} . \\
& 2 \mathrm{C}+\mathrm{O}_{2} \rightarrow 2 \mathrm{CO} \ldots . .
\end{aligned}
$$

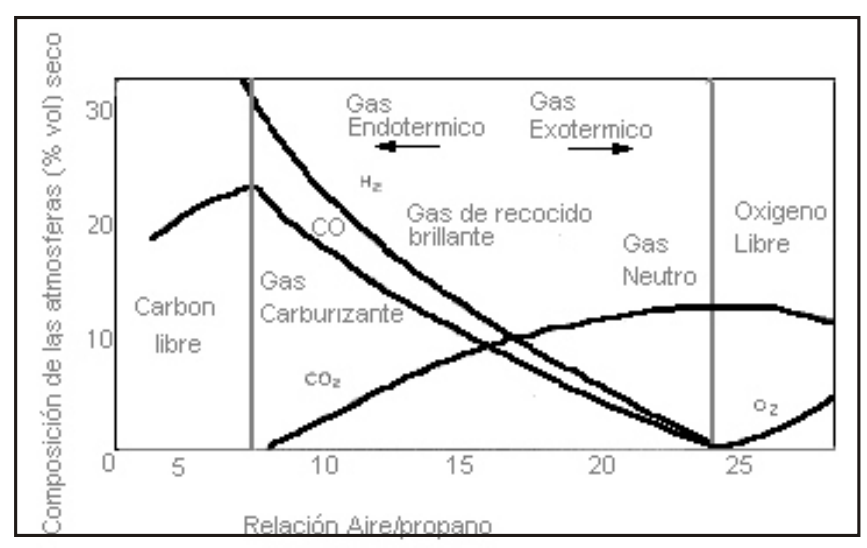

Figura 1. Composición de atmósferas producidas a parti de aire/propano.
Luego de conseguirse la reacción anterior, el gas resultante debe ser rápidamente enfriado en un intercambiador de calor para prevenir un efecto de reversibilidad de las reacciones, formándose Carbono y Dióxido de Carbono a partir del Óxido de Carbono como se representa en la reacción (3).

$$
2 \mathrm{CO} \rightarrow \mathrm{CO}_{2}+\mathrm{C}
$$

La reacción (3) es predominante a temperaturas de 480 a $750^{\circ} \mathrm{C}$.

La mezcla de aire/gas con una proporción en volumen entre 7.1/1 a 7.5/1 debe pasar por el catalizador calentado a una temperatura aproximada entre 980 a $1040^{\circ} \mathrm{C}$, contenido en la retorta a una presión positiva de trabajo. Después de pasar por el catalizador, los gases de reacción son enfriados rápidamente por debajo de $315^{\circ} \mathrm{C}$, previniendo de esta manera la reacción inversa y garantizando una composición adecuada de la atmósfera gaseosa (5).

Para producir mezclas de gases, constituidas fundamentalmente por $\mathrm{H}_{2}$, $\mathrm{CO}$ y $\mathrm{H}_{2}$ y en menores proporciones de $\mathrm{H}_{2}$ y $\mathrm{CO}_{2}$, el propano debe disociarse casi por completo sobre el catalizador, de acuerdo con la reacción:

$\mathrm{C}_{3} \mathrm{H}_{8}+7.2\left(0.21 \mathrm{O}_{2}+0.79 \mathrm{~N}_{2}\right) \rightarrow 3 \mathrm{CO}+5.6 \mathrm{~N}_{2}+4 \mathrm{H}_{2} \ldots \ldots .(4)$

El catalizador más comúnmente utilizado en generadores endotérmicos es un refractario poroso de alta temperatura, como la alúmina, impregnada de Míquel (Ni), para proporcionar una máxima área de superficie de contacto con los gases, produciendo en forma eficiente la reacción (7).

Usualmente el catalizador se instala sobre un difusor, el cual tiene un doble propósito: primero, soportar el peso del catalizador ya que va dispuesto en posición vertical y evitar que éste tapone la entrada de la mezcla aire/gas $y$, segundo, difundir la mezcla de entrada 
homogéneamente sobre todo el catalizador por medio de un plato perforado. Generalmente el tipo de material utilizado para el difusor debe ser no catalítico y bajo en hierro, como la alúmina desactivada $(8,9)$.

\section{DISEÑO Y CONSTRUCCIÓN DEL GENERADOR PROTOTIPO}

Comercialmente existen una variedad de generadores endotérmicos, de los cuales el equipo prototipo desarrollado conserva las características básicas. Los detalles de diseño y construcción y las especificaciones de los componentes se presentan en $(10,11)$ y se compone de los siguientes equipos y accesorios:

1. Unidad de mezcla: Un sistema de distribución con reguladores de presión y caudal.

2. Retorta de calentamiento: construida en acero inoxidable AISI 304 con una placa metálica difusora perforada en el borde inferior, sobre la cual se coloca el catalizador de Míquel.

Temperatura de diseño $=1040^{\circ} \mathrm{C}$, Presión de diseño $=7.4$ psi.

3. Horno de calentamiento: Horno cilíndrico vertical con una potencia calculada de 2100 Watts, para lograr calentar el sistema en 2 horas.

4. Intercambiador de Calor: Diseñado y construido para enfriar el gas endotérmico de 630 a $315^{\circ} \mathrm{C}$ por agua a $33^{\circ} \mathrm{C}$.

5. Compresor: con una presión máxima de 85 psi y con una válvula reguladora manual.

6. Flujómetros: tipo "rotámetros" para medir flujos de aire y propano con escala de 0 a 30 C.F.H y la presión límite admitida es de 30 psi.

7. Un controlador de temperatura marca STM modelo CT/0001 con un nivel de estabilidad de $1^{\circ} \mathrm{C}$. Termocupla: termopar tipo k, cromoalumel y níquel-aluminio, con rango de medición de $-18^{\circ} \mathrm{C}$ a $1260^{\circ} \mathrm{C}$.

8. Manómetros: para medir la presión en cada línea de alimentación gas y aire con un rango de 0 a 15 psi.
El esquema de la figura 2, muestra el montaje completo del sistema, con los equipos seccionados y construidos.

\section{PROCEDIMIENTO EXPERIMENTAL}

Inicialmente se realizó una verificación del funcionamiento de los equipos del sistema, definiendo los parámetros operativos y variables relevantes en el proceso de generación de la atmósfera endotérmica:

1. La relación aire/gas fue de 7.25 para todas las pruebas.

2. Las temperaturas de generación de la atmósfera fueron de 1010 y $1050^{\circ} \mathrm{C}$.

Para el tratamiento térmico de temple $y$ cimentación se definieron los siguientes parámetros y variables de generación:

1. Se trataron 4 muestras de cada uno de los aceros 1018 y 1025 para cada tiempo y temperatura de generación estipulados.

2. La temperatura de sostenimiento fue de 930 'C en todas las pruebas.

3. Los tiempos de mantenimiento de 20,40 , 60 y 120 minutos.

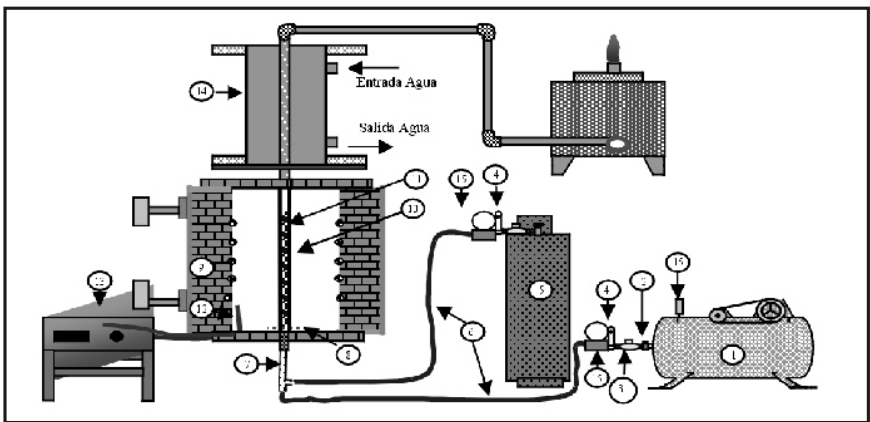

Figura 2. Esquema de la instalación del sistema de atmósfera controlada.

1. Compresor

4. Flujómetro

7. Cámara de mezcla

10. Cámara de calentamiento

13. Controlador

$$
\begin{array}{ll}
\text { 2. Llave de bola } & \text { 3.Regulador de presión } \\
\text { 5. Cilindro de propano } & \text { 6. Tubería de gases } \\
\text { 8. Lamina perforada } & \text { 9. Horno } \\
\text { 11. Catalizador } & \text { 12. Termocupla } \\
\text { 14. Intercambiador } & \text { 15. Manómetro }
\end{array}
$$

4. El medio de temple escogido fue una solución de $10 \%$ de cloruro de sodio en agua (salmuera). 


\section{RESULTADOS Y DISCUSIÓN}

\subsection{Análisis gravimétrico:}

Se determinó la pérdida o ganancia de carbono en el tratamiento térmico de las muestras mediante la medición de la variación de peso de las probetas antes y después del tratamiento para ver la tendencia de protección o descarburación de acuerdo a las variables del ensayo. Se les realizó una limpieza cuidadosa para eliminar depósitos superficiales de óxido, hollín, etc. Se utilizó una balanza analítica marca METTLERTOLEDO, con capacidad de 310 g y una precisión de $1 \mathrm{mg}$.

Los resultados se muestran en las figuras 3 y 4 . Se observó una tendencia a la perdida de peso en el acero 1025, especialmente en los tiempos mayores, mientras que algunas muestras de acero 1018 tuvieron pérdida de peso, pero otras mostraron ganancia de peso, correspondiente a tiempos menores de sostenimiento $y$ temperatura de generación de $1010^{\circ} \mathrm{C}$.

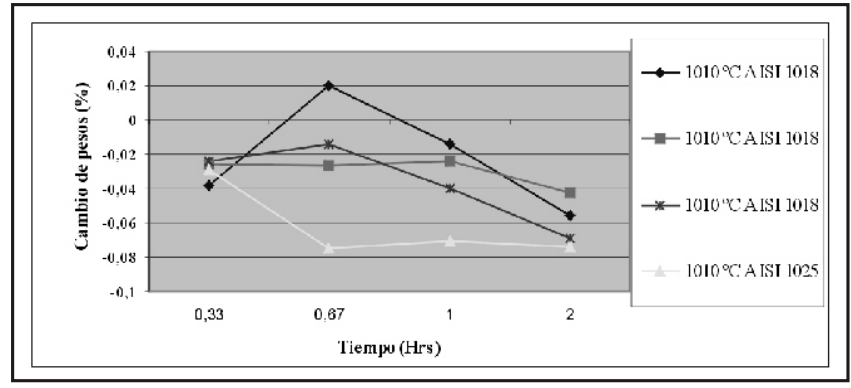

Figura 3. Pérdida de peso en función del tiempo, para los dos aceros a la temperatura de generación de la atmósfera de $1010^{\circ} \mathrm{C}$

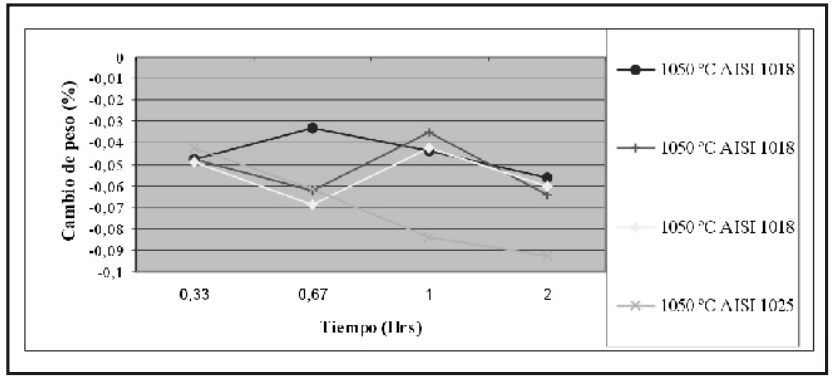

Figura 4. Pérdida de peso en función del tiempo, para los dos aceros a la temperatura de generación de la atmósfera de $1050^{\circ} \mathrm{C}$

\subsection{Análisis de microdureza:}

Se realizaron mediciones de microdureza en la superficie y en el núcleo sobre una sección transversal para cada una de las probetas de ensayo. La medición se realizó según lo especifica la norma ASTM E 384-89 usando un durómetro marca WOLPERT con indentador piramidal Vickers y carga aplicada de $0.1 \mathrm{~kg}$.

Los resultados se muestran en la tabla 1 . Se observó un aumento de dureza en la superficie de algunas probetas de acero 1018, con temperatura de generación de $1010^{\circ} \mathrm{C}$; mientras que las restantes presentaron una tendencia a la pérdida de dureza de la superficie respecto al núcleo. La dureza típica de un acero 1018 en estado normalizado está en el rango de 130 a 150 Vickers.

\subsection{Análisis de Profundidad de Capa:}

En esta medición se utilizó un método metalográfico según la norma SAE J413. Las mediciones se realizaron usando un microscopio óptico marca OLIMPUS BX60, con amplificación hasta 1000X, que contiene una escala de medición. Los resultados se muestran en la tabla 1 .

Se observa un espesor de capa afectada menor en las probetas de acero 1018 para la temperatura de generación de $1010^{\circ} \mathrm{C}$ comparada con las de $1050^{\circ} \mathrm{C}$. Este resultado es consistente con los resultados de pérdida de peso mostrados antes. Mo se observa una correlación clara entre el espesor de capa y el tiempo de sostenimiento en el tratamiento de temple de las probetas.

\subsection{Análisis Metalográfico:}

Se prepararon metalográficamente las probetas en una sección transversal, según lo especifica la norma ASTM E3-95. El análisis microestructural de las muestras preparadas se realizó en un 
microscopio Olimpus BX60, con amplificaciones hasta de 1000X. En las figuras 5 a - d, se muestran las micrografías obtenidas para algunas muestras.

La microestructura típica en el núcleo de las muestras templadas están compuestas principalmente por morfologías de ferrita (áreas claras) y de martensita de bajo carbono (áreas oscuras), con durezas entre 220 y $480 \mathrm{HV}$ para el acero 1018 y entre 330 y 500 HV para el acero 1025 .

La microestructura en la superficie de la muestra de acero AISI 1018, probeta 1C, (Tiempo 20 min., generación a $\left.1010^{\circ} \mathrm{C}\right)$, mostrada en la figura 5(a), no presentó descarburación en la superficie y revela una microestructura de matriz ferritita rodeando las áreas de martensita laminar. La probeta 2, (Tiempo 40 min. Generación a 1010 $\left.{ }^{\circ} \mathrm{C}\right)$, mostrada en la figura 5(b), no presentó evidencia de descarburación y revela una microestructura compuesta de martensita con ferrita en menor cantidad. La probeta 4b (Tiempo 2 hrs. Generación a $\left.1010^{\circ} \mathrm{C}\right)$, mostrada en la figura 5(c) presenta una capa superficial descarburada compuesta de ferrita y martensita/perlita. La probeta 11 (Tiempo $1 \mathrm{hr}$. Generación a $\left.1050^{\circ} \mathrm{C}\right)$, mostrada en la figura 5(d), presentó una capa bastante descarburada compuesta sólo por granos de ferrita. $(11,12)$

Tabla 1. Microdureza y profundidad de capa para los aceros AISI 1018 y 1025 a las temperaturas de generación de 1010 y $1050^{\circ} \mathrm{C}$.

\begin{tabular}{|c|c|c|c|c|c|c|}
\hline \multirow{2}{*}{$\begin{array}{l}\text { Acero } \\
\text { (AISI) }\end{array}$} & \multirow{2}{*}{$\begin{array}{c}\text { Temperatura } \\
\text { Generación } \\
{ }^{\circ} \mathrm{C}\end{array}$} & \multirow{2}{*}{$\begin{array}{l}\text { Tiempo de } \\
\text { Sostenimto } \\
\text { Horas }\end{array}$} & \multirow[b]{2}{*}{ Muestra } & \multicolumn{2}{|c|}{ Microdureza (HV) } & \multirow{2}{*}{$\begin{array}{l}\text { Espesor de } \\
\text { Capa } \\
\text { (Micras) }\end{array}$} \\
\hline & & & & Núcleo & Borde & \\
\hline \multirow{4}{*}{1018} & \multirow{4}{*}{1010} & 0,33 & Probeta 1 & 370 & 478 & 18 \\
\hline & & 0,67 & Probeta 2 & 479 & 552 & 27 \\
\hline & & 1 & Probeta 3a & 571 & 258 & 19 \\
\hline & & 2 & Probeta 4b & 501 & 501 & 12 \\
\hline \multirow{2}{*}{1018} & \multirow{3}{*}{1050} & 0,33 & Probeta 9 & 145 & 120 & 39 \\
\hline & & 1 & Probeta 11 & 145 & 120 & 48 \\
\hline 1025 & & 1 & Probeta 15 & 579 & 450 & 50 \\
\hline
\end{tabular}

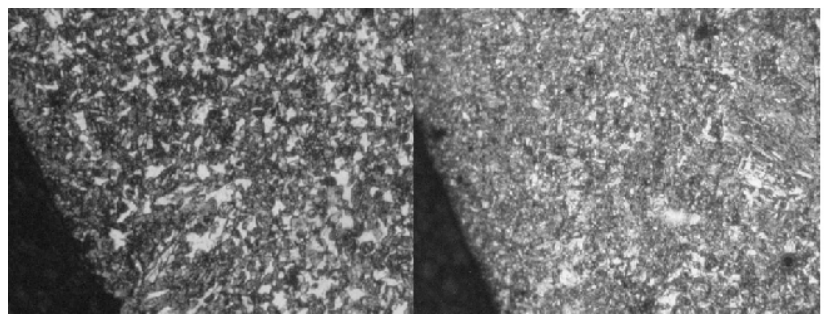

( a ) (b)

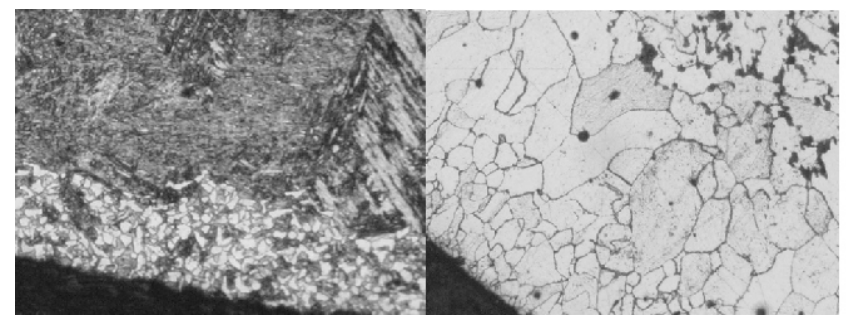

( c ) (d)

Figura 5. Microestructura en la superficie de las probetas de acero 1018.

a) Probeta 1c, (Tiempo 20 min. Generación a $1010^{\circ} \mathrm{C}$ ),

b) Probeta 2, (Tiempo 40 min. Generación a $1010^{\circ} \mathrm{C}$ ),

c) Probeta 4b descarburada (Tiempo 120 min. Generación a $1010^{\circ} \mathrm{C}$ ), y

d) Probeta 11 descarburada (Tiempo 60 min. Generación a $1050^{\circ} \mathrm{C}$ ).

Ataque con Matal 2\%. 100 aumentos. 
Como se desprende de los resultados obtenidos, algunas de las muestras de acero 1018 claramente presentaron cementación y altas durezas en el temple para una temperatura de generación de $1010^{\circ} \mathrm{C}$ y especialmente para tiempos menores de sostenimiento en la cámara del horno de tratamiento térmico, lo cual muestra la potencialidad del equipo para lograr tratamientos térmicos exitosos en atmósferas controladas.

En el caso de las muestras de acero 1025 y especialmente para temperatura de generación de $1050^{\circ} \mathrm{C}$, para tiempos mayores de sostenimiento en el horno, los resultados mostraron una condición típicamente descarburante, debida posiblemente a cambios en la actividad del catalizador, que afecta la composición del gas endotérmico resultante. Por otra parte, los niveles de $\mathrm{CO}_{2}$ medidos fueron relativamente altos generando una atmósfera más oxidante y reductora. Finalmente, la humedad del aire suministrado a la mezcla no se controló mediante filtros.

\section{CONCLUSIONES}

1. Es posible proteger un acero AISI 1018 de la descarburación, o incluso cementarlo en un tratamiento térmico en atmósfera controlada con la ayuda de un sistema generador de gas endotérmico, a través de una dosificación adecuada del propano y aire.

2. La atmósfera obtenida para una temperatura de reacción de $1010^{\circ} \mathrm{C}$ tuvo un efecto endurecedor sobre las propiedades del acero tratado, consiguiendo valores de hasta $483 \mathrm{HV}$ en microdureza y de 27 en espesor de capa para un acero 1018 levemente cementado.

3. El gas endotérmico generado a $1050^{\circ} \mathrm{C}$ produjo la descarburización de las muestras, que se ve reflejado en la pérdida en peso y la menor microdureza obtenidas, debido a un cambio apreciable de la actividad del catalizador especialmente para tiempos mayores de tratamiento.

4. Un control más riguroso de la composición del gas en la atmósfera del horno mediante una medición confiable, directa y continua permitirá tener una operación más confiable del equipo en los tratamientos térmicos de cementación y temple en atmósfera controlada.

5. Una adición de mayor contenido de propano, a través de la relación aire/gas, dentro del horno de tratamiento podría garantizar que la atmósfera sea ligeramente carburante por efecto del enriquecimiento causado a la atmósfera.

\section{REFERENCIAS}

(1) MARTímez, PERES y GORDOM. Teoría y Tecnología del Tratamiento Térmico. Editorial Pueblo y Educación. La Habana 1990. Capitulo 3. Páginas 46-52, 102-113.

(2) ASM HAMDBOOK. Heat treating. Volumen 4. 1997. Páginas 312-324, 454-461.

(3) MEMEMYI REZSO. Controlled Atmospheres for Heat Treatment. Editorial Pergamon Press. Primera edición. 1984. Paginas 46, 22-23, 70-71, 186-198.

(4) ASTIM SEMA. Manual Práctico de Fraccionador Lindberg. Centro de información tecnológica.

(5) ZAHMISER JAMES. Materials Engineering Institute. MEI. ASM international. Curse 10. Páginas 19-21.

(6) AVNER SYDNEY. Introducción a la Metalurgia Física. Ediciones Mc Graw Hill. Segunda edición. 1979. Páginas 252-284, 317-323.

(7) VALEMCIA ASDRUBAL. Tecnología del Tratamiento Térmico de Metales. Editorial Universidad de Antioquia. Segunda edición. 1992. Páginas 344-346, 608-612. 
(8) APRAIZ BARREIRO. Tratamiento Térmico de los Aceros. Editorial Dossat. Octava edición, Madrid España. 1965. Páginas 161-163.

(9) DROGUETT SERGIO. Elementos de Catálisis Heterogénea. Monografía Mo 26. Secretaria general de la organización de los Estados Americanos. Washington. D.C. 1983. Páginas 33-42.

(10)HOTCHKISSS WEBBER. Protective Atmospheres. Jhon Wiley and Sons, inc. Mew York. 1953. Páginas 33, 101-105.

(11)CASTRO IRVIM. Diseño e Implementación de un Horno Piloto de Atmósfera Controlada con base a una mezcla Propano-Aire para Tratamientos Térmicos. Tesis de grado. Universidad del Valle. 2003. Páginas 5-9, 43-45.

(12)PAZ HURTADO JOSÉ HARIMSOM. Diseño y Construcción de un Generador de Gas Endotérmico Prototipo para Tratamiento Térmico de Atmósfera Controlada. Tesis de Grado. Universidad del Vale. 2004. Pág. 24$25,36-37,42-48,75,93-94$. 